\title{
Consequences of the provision of laboratory services for the National Health Service by commercial firms: a view from the University sector
}

\author{
L G Whitby, C C Bird, J G Collee, W N McDicken
}

\section{Introduction}

The long standing arrangements whereby laboratory diagnostic services for most patients in British hospitals or general practice have been provided by NHS or university departments are being questioned by some politicians and NHS administrators. Before the alternative method of provision by commercial firms is adopted for whatever reason it is important to consider the possible and foreseeable adverse consequences for the NHS and United Kingdom medical schools.

One of the clearest and most comprehensive descriptions of the functions that hospital laboratory services seek to fulfil is contained in $\operatorname{HM}(70) 50 .{ }^{1}$ Much of what was stated there remains true today, provided that the organisational terminology is updated. Although $\mathrm{HM}(70) 50$ related to England and Wales, the principles described in paragraph 2 were accepted as being generally applicable elsewhere in the United Kingdom.

In centres with medical schools hospital laboratories (whether staffed by universities or the NHS) have for many years acted as focal points for the provision of diagnostic services and related specialist advice. They have fulfilled important educational roles and have been active in performing or supporting much research and development work that has been fundamental to advances in medical practice and is of direct relevance to NHS interests.

University of

Edinburgh and the

Royal Infirmary of Edinburgh

Department of

Clinical Chemistry

L G Whitby

Department of

Pathology

C C Bird

Department of

Bacteriology

J G Collee

Department of

Medical Physics and

Medical Engineering

W N McDicken

Correspondence to:

Professor L G Whitby,

Chemistry, The Royal

Infirmary of Edinburgh

EH3 9YW, Scotland

Accepted for publication

16 January 1990

\section{Future laboratory services}

Medical practice can be expected to depend increasingly on diagnostic services that are readily available, reliable, sensitive and specific, reasonably comprehensive and up to date. In view of the growing complexity of medicine, with its various programmes of postgraduate education and training leading to accreditation in a wide range of specialties, staff in diagnostic laboratories can expect to be called on more and more for advice. Much of this advice can best be provided by personal consultation between laboratory and clinical staff in hospital or general practice. Laboratory staff already have increasingly key roles in therapeutics-for example, in the planning of radiotherapy, ultrasonography, drug monitoring and as members of total parenteral nutrition teams and providers of on-site frozen section services.
There will probably be an increasing need for the rapid provision of laboratory investigations close to patients-for example, in intensive care units, accident and emergency departments, hospital outpatient departments and in general practice surgeries. The growing range of analytical equipment described as suitable for use by non-laboratory staff close to the patient will probably lead to more decentralisation of some investigations, a development that will call for daily equipment maintenance to be provided locally, and for objective quality control procedures, as well as for local education programmes which will have to be periodically repeated. Many of the problems that arise from the use of "blackbox" equipment in wards and clinics are due to staff changes, such as the introduction of new staff, and the frequency with which problems can and do occur means that satisfactory back-up arrangements are required nearby.

The NHS laboratory services, based as they are in most major hospitals, are well placed to meet present and the likely future demands for investigations and high technology services, given the necessary resources. Nonurgent requirements, however, can be provided from off-site facilities, in some cases located many miles (even hundreds of miles) away, as long as response times are satisfactory. Tendering for these services might lead to more cost effective provision in some parts of the NHS, especially where NHS laboratories are providing a purely commercial type of service virtually identical with that available outside the NHS. Examples could include the following:

\section{NON-URGENT CLINICAL CHEMISTRY}

Specimen preparation facilities will still be required locally, and laboratory staff and equipment will still be needed locally, especially in acute general hospitals, to perform some of the same investigations whenever results are required urgently.

\section{NON-URGENT HISTOPATHOLOGY}

Some elements of cervical cytology, such as routine screening of patients in general practice, provide one example of this. There will still, however, need to be satisfactory call and recall arrangements, and local provision will be required for investigating abnormal results and carrying out follow up. 


\section{NON-URGENT VIROLOGY}

Screening programmes for human immunodeficiency virus (HIV), hepatitis $B$, and rubella could be put out to tender-for population based studies. For individual patients, however, a local service would still be needed because results may be required urgently and consultation with laboratory staff is often necessary.

\section{EQUIPMENT SERVICING}

A cost effective service for the maintenance of medical equipment by an off-site organisation can be provided as long as response times are adequate. For acute general hospitals, however, enough back-up equipment needs to be held on site or close by, together with staff capable of operating the local service.

These examples indicate that any cost reductions that might at first sight stem from transferring components of the laboratory services off site to commercial firms are liable to be eroded by the continuing need to provide some form of service locally. Indeed, such necessary duplication of provision might mean that the total cost to the NHS - that is, the cost of a commercial service plus the corresponding NHS-provided componentwould be increased rather than decreased. Also, with these and other possible examples of services provided at a distance, commercial laboratories might have great difficulty in providing a satisfactory consultant advisory service.

Any approach to competitive tendering for components of the laboratory based services should take into account: (1) the quality of the services to be provided to NHS patients; (2) opportunities for cost reduction in relation to the existing pattern of service provision; and (3) the ability of the present type of service and any commercial alternative to provide both for foreseeable and unforeseen changes in requirements for diagnostic services.

\section{Quality of service}

A narrow interpretation of quality would focus attention on analytical reliability. Adverse comparisons might be made between the voluntary and confidential participation of NHS and university laboratories in external quality assessment (EQA) schemes and the apparently obligatory involvement of members of the Association of Independent Pathology Laboratories in the same or equivalent EQA schemes; results for the latter are said to be open to inspection. Such comparisons would be of questionable validity as very nearly all NHS and university laboratories participate in the national (UK) EQA schemes, and one very good reason for maintaining confidentiality of results is to obviate the otherwise natural tendency to treat EQA specimens with special care. There are other aspects of quality of the diagnostic service that need to be taken into account, in addition to analytical reliability, reflected in the following ${ }^{2}$ :

"The practice of pathology is a medical specialty essential to patient care. The pathology service shall include, but not be limited to, the examination of clinical specimens, interpretation, consultation, appropriate scientific investigation and education in the prevention, recognition, diagnosis, and treatment of disease."

In the context of this the importance of the laboratory disciplines in medical audit should be recognised. This includes participation in clinicopathological conferences when clinical diagnoses and the results of treatment are subjected to close scrutiny, as well as the final search for proof of the correctness of clinical diagnosis and management afforded by properly conducted necropsies. Were these laboratory activities to be lost as a result of transfer of services to some distant commercial organisation, the quality of medical care would suffer.

\section{Cost reduction}

Most hospital laboratory departments operate within predefined budgets. This is in spite of their usually having no direct control over clinical demand for the investigations they perform. Departments that have been required to make savings have mostly succeeded in doing so without obvious deterioration as yet in their performance in EQA schemes. This has been at the expense of increasing strain on all categories of staff, however, and, in the case of university departments, erosion of their staff's ability to fulfil their obligations to engage in teaching and research.

Cost reductions should be achieved by reducing the number of requests for laboratory investigations, but this requires collaboration between clinicians and laboratory staff, involving discussion and education; such collaboration might be difficult to achieve with staff in commercially provided pathology services. With present arrangements there is a common objective to minimise costs to the NHS, but this common objective would be absent in commercial services for which the objectives normally include maximising of profits. Instead of mutual cooperation, administrative fiat would probably be needed in order to cap NHS expenditure on private pathology services. This could have serious medicolegal implications for NHS authorities.

Commercial provision of laboratory services does not necessarily spell cost reductions and financial savings. For instance, NHS management would incur costs attributable to the monitoring arrangements needed to ensure that the commercial laboratories were fulfilling the requirements specified in their contracts to provide certain diagnostic services. These costs would have to be offset against any savings that management might otherwise claim to have made by engaging commercial firms.

\section{Changing requirements}

As arrangements now stand, it is easy for pathology staff and clinicians to discuss likely future needs for diagnostic services on a professional basis, and for their financial implications to be presented to NHS management for con- 
sideration as part of forecast estimates each year. It might be difficult to make equivalent arrangements for the developing needs of the NHS in respect of pathology and medical physics services in those cases where these services are to be provided by commercial firms.

Some changes in NHS requirements for pathology services cannot be readily foreseen, and some may occur suddenly. For instance, the prompt and thorough investigation of an outbreak of infectious disease in a hospital or in the community can incur costs that are not readily predictable. It is hard to see how arrangements that depend on privatised pathology services could be expected to respond quickly to such unforeseen emergencies, unless financial considerations could then be suddenly disregarded by the NHS authorities and the conditions of a previously costed commercial contract varied.

\section{The pathological specialties and medical education}

Under the terms of the Medical Act 1983, chapter 54, the education committee of the General Medical Council has the general function of promoting high standards of medical education and coordinating all stages of medical education. The committee made several direct or indirect references to the clinical sciences (laboratory medicine) notably in paragraphs 13-14, 35, and 47-48 of its Recommendations on Basic Medical Education. ${ }^{3}$

The specialties that comprise pathology are fundamental to the undergraduate teaching of clinical medicine. To fulfil their responsibilities teaching staff require ready access to a wide range of specimens and related clinical information to provide essential teaching material and to keep themselves up to date and active in research, so as to be in a position to teach the doctors of the future properly. The expectations of the GMC education committee will be difficult for medical schools to meet wherever substantial components of the pathological specialties are no longer the responsibility of clinical academic or NHS consultant staff, because a commercial organisation might act independently of the medical school's interests. Indeed, we forecast that the committee will have to consider whether it should continue to recognise any medical school that has its teaching arrangements for medical students disrupted in this way.

The GMC education committee can be expected to take a correspondingly serious view of the effects on postgraduate medical education if important components of the pathological specialties are transferred away from the laboratories at present responsible for providing them. The committee referred to the provision of adequate laboratory facilities, ${ }^{4}$ and the Joint Committees on Higher Training made similar stipulations in respect of facilities to be provided in hospitals seeking to be recognised as suitable for postgraduate training purposes. Any substantial change in the way in which pathology services are provided to the NHS seems likely to lead to reviews of the continuing suitability of posts previously recognised for postgraduate training in those specialties for which these committees are responsible.

To obviate the danger of withdrawal of GMC approval for undergraduate medical education, or loss of recognition for postgraduate training of potential specialists, medical schools and NHS authorities might find themselves compelled to retain enough local access to a range of pathological material sufficient to enable them to fulfil their educational commitments. The laboratory staffing and facilities that this would entail would spell duplication of financial commitment and might increase the overall cost. It would also introduce the possibility of dual standards of performance, one local (NHS) and the other commercial.

We cannot emphasise too strongly that all the specialties that comprise the university pathology departments require full and ready access to specimens obtained from patients to fulfil their obligations to undergraduate and postgraduate medical education. If important components of the diagnostic service are separated off the basic critical mass of these laboratories is liable to be damaged. The educational and the diagnostic service activities of pathology departments depend on their having this basic foundation.

\section{Recognition of hospital laboratories for the training of laboratory staff}

Pathology departments seeking to be registered for the in-service training of staff are required to meet standards that have been set by the relevant professional bodies. The various bodies involved, such as the Royal College of Pathologists in respect of trainee pathologists and the Medical Laboratory Technicians (MLT) Board of the Council for Professions Supplementary to Medicine for trainee medical laboratory scientific officers (MLSO), have established their particular requirements.

The NHS and university laboratory departments mostly appoint staff who they proceed to train themselves, or recruit staff who have previously worked in other similar departments that are registered for training purposes. If important components of the diagnostic service which these departments provide are transferred to commercial laboratories their registration as training departments will presumably have to be reviewed and may in some cases be withdrawn. Relatively few commercial laboratories are registered for the training of MLSO and very few or none for the training of pathologists.

\section{Research and service developments and} the laboratory specialties

The NHS has benefited in many ways over the years from research and development work undertaken in laboratory departments staffed by university or NHS personnel. The evidence can be found in this and related special journals, the Lancet, and the British Medical Jour- 
nal, and a wide range of specialist clinical publications.

Examples of research and development work that have contributed to improvements in NHS provision and practice include investigations concerned with hepatitis B and HIV diagnostic serology; rigorous assessment and evaluation of new laboratory equipment on behalf of the United Kingdom health departments before its approval for general purchase; the introduction and subsequent operation of external quality assessment schemes covering most laboratory disciplines and a wide range of analytes; the development of diagnostic techniques such as percutaneous renal biopsy and liver biopsy and the subsequent training of staff nationwide so that these procedures are now regarded as commonplace; and the development of automated analysis of electrocardiogram signals which allow ambulant patients to be monitored over prolonged periods.

Important as the foregoing examples of laboratory based research contributions have been for improving the quality of the service to patients provided by the NHS, pathology and medical physics departments in teaching hospitals and elsewhere in the NHS have fulfilled essential roles as supports for a wide range of clinical research. For instance, much of the research work undertaken in teaching hospitals by university departments of medicine, obstetrics and gynaecology, paediatrics, and surgery could not have been done properly if there had not been close collaboration with these departments. Such combined clinical and laboratory research will probably be more difficult to arrange, and indeed might no longer be feasible, wherever the mainstream laboratory services are provided from a distance by commercial organisations. Even minor advances in treatment or management of patients may require substantial changes or improvements in laboratory services, which are only readily obtainable from departments that are themselves committed to evolution and to service developments. This summary description applies to NHS laboratories just as much as to university departments.

As far as university pathology and medical physics departments are concerned, transfer to commercial firms of important components of the diagnostic services they presently provide for the NHS would be at variance with the Government's stated aims ${ }^{6}$ : "The Government is firmly committed to maintaining the quality of medical education and research. It recognises the complexity and special needs of these areas..."

\section{Conclusion}

The threat to the future provision of diagnostic pathology services was seen to be so great in 1987 that the then president of the Royal College of Pathologists sought and obtained a written assurance from the then Secretary of State for Social Services that the profession would be consulted before steps were taken with a view to the possible privatisation of pathology services. The president of the Royal
College of Pathologists and the president of the Association of Clinical Pathologists later referred to the Secretary of State's reply when making the following statement, ${ }^{7}$ which we unreservedly endorse:

"We feel that all pathologists should realise the importance of maintaining an integrated analytical and consultative service and the importance of maintaining both the standards of pathology and the standards of many aspects of the hospital service which depend upon the continuation of integration of pathology services within the hospital sector. Emphasis should also be placed on the importance of this integrated pathology service in the training of both medical and non-medical staff, in the maintenance of high standards of teaching and research, and in the provision of a link between the hospital and general practitioner services. It is difficult to appreciate how local privatisation can meet all of these objectives and it is therefore suggested that if any pathologists are confronted with a local initiative towards privatisation it is imperative that they should become involved in the preparation of the specification of private tenders and that physicians and surgeons should also be given an opportunity to comment and contribute. Pathologists should ensure that the Secretary of State's assurances on the maintenance of standards are respected. They should not condone the separation of the analytical from the consultative aspects of the service."

Rather than proceed with a programme of competitive tendering and possible privatisation of part or all of a health authority's laboratory services, heads of departments (university and NHS) with responsibilities for providing these services should review their present working arrangements. They should then discuss these arrangements with their colleagues in geographically related departments and with the relevant administrative and financial authorities, with a view to defining the future requirements for the service. This will involve preparing detailed specifications of the services to be provided and a review of the various ways in which these requirements might be met, so as to determine the most cost effective and professionally acceptable arrangements. Consultation with hospital clinical staff and with representatives of local general practitioners will be an essential component of this process and should be undertaken before final decisions are made.

$1 \mathrm{HM}(70) 50$. Hospital laboratory services. London: Her Majesty's Stationery Office, 1970.

2 College of American Pathologists. Standards for laboratory accreditation. Skokie, Illinois: College of American Pathaccreditation.
ologists, 1988.

3 General Medical Council: Education Committee. Recommendations on basic medical education. London: General Medical Council, 1987.

4 General Medical Council: Education Committee. Recommendations on the training of specialists. London: Genera Medical Council, 1980.

5 Royal College of Physicians of London: Joint Committee on Higher Medical Training. Training Handbook 1988/89. London: RCP, 1989:12.

6 Working for patients. Paragraph 4.30. London: Her Majesty's Stationery Office, 1989.

7 Pennington GW, Williams ED. Pathology privatisation initiatives in the public sector. ACP News 1988; 10. 